\title{
Sobre el régimen legal aplicable a la protección ambiental del Océano Ártico
}

\author{
Giovanny Vega Barbosa* \\ Sergio Diaz Rodríguez**
}

\begin{abstract}
RESUMEN
Las particularidades del medio ambiente Ártico han propiciado un importante debate académico en relación con la suficiencia de su régimen legal internacional actual para garantizar una protección adecuada. Al respecto, se evidencia una enorme tensión entre aquellos que defienden la idoneidad de la Convención de Naciones Unidas sobre el Derecho del Mar como herramienta efectiva para encauzar los esfuerzos de protección, y aquellos que destacan que las especialisimas necesidades del Ártico no pueden ser adecuadamente abordadas bajo la égida de este instrumento. Los autores abogan por una protección efectiva a partir de la utilización eficiente de los instrumentos disponibles y sobre la base de la cooperación.
\end{abstract}

Océano Ártico - medio ambiente - Consejo Ártico

\section{About the legal regime apllicable to the environmental protection of the artic ocean}

\begin{abstract}
The particularities of the Arctic environment have encouraged a strong debate over the sufficiency of its current legal regime to guarantee an adequate and sufficient protection. In this context, a strong tension is discernible between those who sustain that protection efforts over this area can be effectively channeled through the United Nations Convention on the Law of the Sea, and those who believe that the very special needs of the Arctic cannot be addressed under such a general instrument. The authors advocate for an effective protection through the efficient utilization of the available resources, and on the basis of cooperation.
\end{abstract}

$$
\text { Arctic Ocean - environment - Arctic Council }
$$

\footnotetext{
* Giovanny Vega Barbosa, Profesor Principal de la Universidad del Rosario en las cátedras de Teoría del Derecho Internacional y Derecho Internacional Público. Correo electrónico: giovanny.vega@urosario.edu.co.

** Sergio Andrés Díaz Rodríguez. Especialista en Derecho del Medio Ambiente de la Universidad Externado de Colombia. Correo electrónico: diaz.sergio@ur.edu.co.

Este trabajo se hace parte de los resultados obtenidos dentro del trabajo del Grupo de Investigación sobre Derecho del Mar del Colegio Mayor de Nuestra Señora del Rosario.

Artículo recibido el 9 de agosto de 2015 y aceptado para su publicación el 30 de agosto de 2016.
} 


\section{INTRODUCCIÓN}

$\mathrm{L}$ as discusiones en relación con la necesidad de una regulación legal vinculante y diferenciada para la protección medioambiental del Ártico constituyen el objeto de estudio del presente escrito. Nuestra investigación se dirigió a determinar si, a la luz de la especial vulnerabilidad del medio ambiente ártico, la normativa vigente constituye una base suficiente de protección que permita aproximarse de forma efectiva a sus problemáticas. A su turno, la noción de "suficiencia" se evaluó en función de la capacidad de la normativa actual para vincular de manera efectiva a los Estados árticos frente a los estándares ambientales deseados, y de la especificidad de sus contenidos como garantía de una aproximación útil frente a la especial vulnerabilidad de los ecosistemas árticos ${ }^{1}$.

Nuestra investigación pudo constatar la existencia de dos vertientes doctrinales claramente diferenciables. De una parte, aquellos autores que defienden la suficiencia del régimen legal general previsto en la Convemar, a su vez complementado por ciertos instrumentos de carácter regional y multilateral. Los tratadistas que defienden lo opuesto lo hacen en dos dimensiones principales. La primera, destaca que la regulación actual dispone de un carácter genérico y, por tanto, no responde a las especiales características del medio ambiente ártico; la segunda, pone de manifiesto que la normativa específica existente está constituida por instrumentos de carácter no vinculante, por lo que carece de la entidad adecuada para vincular a los Estados a los estándares de protección y conservación medioambiental que se estiman necesarios.

El presente trabajo se compone de tres partes principales. En la primera parte se efectúa la caracterización del Ártico como espacio. El propósito no es otro que dar a conocer las especiales condiciones y fragilidad de esta zona. En la segunda parte se analiza el régimen legal aplicable a la protección medioambiental del Ártico, así como las discusiones doctrinales acerca de su suficiencia. La tercera parte es conclusiva y contiene las propuestas de los autores en relación con la problemática sub examine.

${ }^{1}$ La doctrina coincide en señalar que, desde la óptica geográfica, solo Rusia, Estados Unidos de América, Canadá, el reino de Dinamarca y el reino de Noruega, constituyen Estados árticos en estricto sentido. Lo anterior, por ser estos los que disponen de salientes costeros con proyección al Océano Ártico. Sin perjuicio de lo anterior, Finlandia, Suecia e Islandia disponen de voz y voto en las decisiones que conciernen a la protección medioambiental de los ecosistemas árticos y, por esta razón, de la denominación de Estados árticos para esos efectos. Al respecto ver: Shusterich, K., "International Jurisdictional Issues in the Arctic Ocean”, Ocean Development E International Law, Vol. 14/3, 1984, pp. 237; Feder, B., 'A Legal Regime for the Arctic', Ecology Law Quarterly, Vol. 6/4, 1978, pp. 801-809; Lakhtine, W., "Rights Over the Arctic", American Journal of International Law, Vol. 24, 1930, pp. 703. 


\section{El Ártico COMO ESPACIO Y SU ESPECIAL VULNERABILIDAD FRENTE A LA DEGRADACIÓN AMBIENTAL}

\section{Caracterización geográfica, física y económica}

Explica Miryam Colacrai de Trevisan que la locución Arktis, de la palabra griega Arktos (oso), era utilizada como concepto astronómico relacionado con la constelación Osa Mayor, y aludía a la región del Norte donde el sol no se pone en verano ${ }^{2}$.

Al aludir al Ártico nos referimos al espacio geográfico que rodea el Polo Norte geográfico del planeta ${ }^{3}$, espacio que comprende tierras y hielo y, que al igual que su referente sureño, tiene la forma de un casquete esférico. Evidencia científica que data desde 1976 indica que la característica principal del Ártico es que su superficie está cubierta de hielo hasta en $90 \%{ }^{4}$.

Se trata de una zona de un inmenso potencial económico. En efecto, aunque en el Ártico habita menos del $1 \%$ de la población mundial, contiene entre el 10 y $15 \%$ de los recursos mineros y energéticos del planeta ${ }^{5}$. Se estima que en el Ártico existen reservas de más de 1.669 trillones de metros cúbicos de gas natural. El potencial en hidrocarburos se valora en un trillón de dólares solo en la porción del Ártico de los Estados Unidos, y de 1,7 trillones de dólares en el Ártico ruso ${ }^{6}$. Asimismo, se calcula que los bancos de pesca comercial más grandes del globo se hallan en esa región ${ }^{7}$.

Históricamente los Estados con proyección hacia el Ártico implementaron diferentes modelos económicos de explotación. El modelo ruso se caracterizó por una acción muy agresiva y económicamente ambiciosa con miras al desarrollo industrial de sus territorios en Siberia. Combinó una alta inversión económica con una presencia poblacional importante. De otra parte, el modelo económico de Estados Unidos de América se concentró en la extracción con una mínima presencia de población y baja inversión en infraestructura. Exempli gratia los 4.373 habitantes de la ciudad de Barrow en Alaska contrastan con los 561 billones de dólares generados por la extracción de petróleo hasta el $2012^{8}$.

${ }^{2}$ Colacrai de Trevisan, M., El Ártico y la Antártida. Su rol en las relaciones internacionales. Su relevancia desde la perspectiva ambiental, Centro de Estudios en Relaciones Internacionales de Rosario (CERIR) y Centro de Estudios Canadienses de Rosario (CECAR), 1998, p. 10.

${ }^{3}$ Marqués, E, "La condición jurídica del Ártico y la Antártica: un asunto pendiente en la agenda jurídico-política de las relaciones internacionales contemporáneas”, Revista de Relaciones Internacionales de la UNAM, No 107, mayo-agosto 2010, pp. 43.

${ }^{4}$ Thorén, R., Picture Atlas of the Arctic, Elsevier Science Ltd, 1969, pp. 7

${ }^{5}$ Encyclopedia of the Arctic, 2005, Vol. 1, pp. 531.

${ }^{6}$ Conley, H., Arctic Economics in the $21^{\text {st }}$ Century. The Benefits and Costs of Cold, Center of Strategic \& International Studies, Washington D.C, 2013, pp. 2-3.

${ }^{7}$ Marqués, E., op. cit., pp. 44.

${ }^{8}$ National Energy Technology Laboratory, Alaska North Slope Oil and Gas: A Promising Future or an Area in Decline?,2009, <http://www.boem.gov/uploadedFiles/BOEM/Oil_and_Gas_Energy_Program/ Resource_Evaluation/Reserves_Inventory/2009DOENorthstarPotential.pdf.> 


\section{El frágil ecosistema Ártico: peligros derivados de la contaminación}

El Océano Ártico alberga una inmensa biodiversidad y se constituye como el área silvestre más grande y abundante del hemisferio norte. Esto la convierte en una zona de interés tanto para la investigación científica como para la explotación industrial. Sin embargo, las actividades industriales y la construcción de numerosas infraestructuras de explotación han sido generadoras de serios problemas de contaminación y polución en la zona debido a la deposición de contaminantes 9 . En el Ártico encontramos minas para la extracción de diamantes, complejos para la recolección de madera, estrategias para la sobrepesca y propuestas para la extracción submarina de hidrocarburos. De igual forma, es posible verificar la instalación de tuberías en alta mar y la expansión de la industria con el ideal de construir plantas nucleares ${ }^{10}$.

La importante actividad industrial en el Ártico no se compadece con el nivel de entendimiento frente a las reales consecuencias de la contaminación en este espacio y respecto de las medidas para mitigar sus impactos. Debido a lo anterior, en 1972 dos investigadores del Instituto Tecnológico de Massachusetts llevaron a cabo estudios acerca de las consecuencias del vertimiento de petróleo en el hielo marino ${ }^{11}$. Las investigaciones concluyeron que el petróleo atrapado en las capas de hielo fungía como aislante térmico protegiendo al hielo del aire frío y previniendo de esta forma la consolidación de nuevas capas de hielo ${ }^{12}$, una situación que por sí misma propicia el descongelamiento. Igualmente, se ha observado que cualquier sustancia atrapada en el hielo que sea más oscura que este reduce el porcentaje natural de reflexión, incrementando el coeficiente de absorción de calor $^{13}$. Hace dos años la Guardia Costera de Estados Unidos había efectuado experimentos mediante el derrame de 55 galones de petróleo en un bloque de hielo en Alaska, comprobando así que el petróleo se adhería a las capas de hielo ${ }^{14}$. Sin embargo, el mecanismo que determinaba la adherencia del petróleo al hielo no pudo ser explicado ${ }^{15}$.

Sin perjuicio de lo anterior, el derretimiento del hielo propicia la presencia de más agentes contaminadores en el Ártico a causa de la apertura de nuevas rutas de navegación. Al seguir las mismas corrientes de agua que sigue la fauna ártica, los derrames de crudo en el ártico constituyen una gran amenaza a la biodiversidad marina. Esto es

\footnotetext{
${ }^{9}$ Nowlan, L., Arctic legal regime for Environmental Protection, International Union for Conservation of Nature and Natural Resources, Gland, Cambridge and Bonn, 2001, p. 14.

${ }^{10}$ Nilsen, T, "Floating Nuclear Plants in the Siberian Arctic?", World Wildlife Foundation, Arctic Bulletin 4.00, 2000.

${ }^{11}$ Wolfe, L. Stephen y Hoult, David P., "Effects of Oil under sea ice", Journal of Glaciology, Vol. 13/69 (1974), pp. 492.

12 Wolfe, L. y Hoult, D., op. cit., pp. 492.

${ }^{13}$ Feder, B, op. cit., pp. 793.

${ }^{14}$ Vance, G.P., Control of Arctic Oil Spills, Ocean Industry, Washington, 1971, pp. 14; Wolfe, L. y Hoult, D., "Effects of Oil under sea ice", Journal of Glaciology, Vol. 13/69, 1974, pp. 11.

${ }^{15}$ Feder, B., op. cit., pp. 793.
} 
particularmente grave pues la cadena alimenticia en el Ártico es más corta y por tanto más vulnerable a un evento de contaminación ${ }^{16}$.

\section{La relación especial de los pueblos del Ártico con su entorno}

La presencia de poblaciones humanas en el Ártico obedeció históricamente al derretimiento del hielo, especialmente como consecuencia de la apertura de espacios de agua para que los pescadores y cazadores extendieran sus faenas y campañas de caza ${ }^{17}$.

De esta forma, se consolidaron grupos humanos como los lapones, samoyedos, ostiacos, tunguses, aleutianos y esquimales. Esta situación permite diferenciar el Ártico de la Antártica. En efecto, en la región ártica se han consolidado grupos humanos con actividades económicas de alto grado de estabilidad, entre ellos, la ciudad de Murmansk en Rusia con 470.000 habitantes y la provincia de Norrbotten en Suecia con 265.000 habitantes.

Algunas de las actividades económicas de las comunidades más antiguas del Ártico perviven hasta hoy, entre ellas, la cría de renos por parte de los Lapps en Siberia. Estas poblaciones dependen de esta actividad, razón por la que son especialmente vulnerables a problemas ambientales que afecten los pastizales. La doctrina documenta la transformación de las actividades económicas tradicionales por prácticas industriales que a su vez han afectado la salud de la población debido a la introducción de nuevos contaminantes ${ }^{18}$.

\section{RÉGIMEN LEGAL PARA LA PROTECCIÓN MEDIOAMBIENTAL DEL OCÉANo Ártico}

Linda Nowlan explica que el medio ambiente ártico se ve afectado por los fenómenos mundiales de cambio climático y la deposición de contaminantes que emanan de todas partes del globo y que se transportan por la atmósfera. Aunado a lo anterior, los principales problemas a nivel local resultan de las crecientes actividades de extracción de recursos, la minería, los ejercicios militares y la industria turística ${ }^{19}$. No obstante, a diferencia de lo que ocurre en la Antártica, no existe un sistema de protección ambiental jurídicamente vinculante para el Océano Ártico. Son entonces las legislaciones domésticas de los Estados árticos las que determinan las actuaciones relevantes. Más allá de la normativa interna, el régimen de protección ambiental del Ártico está constituido por normas sin una naturaleza jurídicamente vinculante y un foro de discusión

\footnotetext{
${ }^{16}$ Ministry of Supply and Services, the Mackenzie Valley Pipeline Inquiry (1977), pp. 72.

${ }^{17}$ Según lo explica Miryam Colacrai de Trevisan, entre los especialistas se estima que la mayor migración en el Ártico debió tener lugar entre 5000 y 6000 años atrás, los períodos más calientes de la actual era interglaciar. Ver Colacrai de Trevisan, M., op. cit., p. 15.

${ }^{18}$ Stepens T. \& VanderZwaag David L. (Eds.), Polar Oceans Governance in an Era of Environmental Change, Northampton, Edward Elgar, 2014, p. 48.

${ }^{19}$ Nowlan, L., Arctic legal regime for Environmental Protection, International Union for Conservation of Nature and Natural Resources, Gland, Cambridge and Bonn, 2001, p. 3.
} 
y concertación, el Consejo Ártico, prima facie sin personería jurídica internacional ${ }^{20}$. Sin perjuicio de lo anterior, la doctrina reconoce que las disposiciones nacionales se han ajustado periódicamente en función de tratados para la protección internacional de los océanos y de sus recursos vivos ${ }^{21}$. Asimismo, se constata una tendencia creciente a la celebración de acuerdos bilaterales vinculantes entre los Estados árticos así como iniciativas de cooperación en aspectos claves ${ }^{22}$. De otra parte, cada vez son más las voces a favor de la Convención de Naciones sobre el Derecho del $\mathrm{Mar}^{23}$ y normativas en el ámbito multilateral como herramientas útiles y suficientes para afrontar las principales preocupaciones medioambientales del Océano Ártico.

\section{La Convención sobre el Derecho del Mar y la Organización Maritima Internacional}

Conforme a la Convemar, los Estados costeros árticos disponen de soberanía en la franja correspondiente al mar territorial, derechos de jurisdicción en una zona económica exclusiva de hasta 200 millas náuticas contadas desde las líneas de base desde las cuales se mide la anchura del mar territorial, y derechos soberanos en la plataforma continental hasta el borde exterior del margen continental ${ }^{24}$. Más allá de los espacios de jurisdicción nacional el Océano Ártico forma parte del Alta $\mathrm{Mar}^{25}$.

Sin perjuicio de lo anterior, las especiales condiciones del Océano Ártico no fueron tenidas en cuenta de manera particular durante la Tercera Conferencia sobre el Derecho del $\mathrm{Mar}^{26}$. Por esta razón, no sorprende que la protección ambiental de las denominadas “Zonas cubiertas de hielo" corra por cuenta de una única disposición, a saber, el Artículo 234. Esta norma faculta a los Estados para emitir y hacer cumplir leyes con el propósito de prevenir, reducir y controlar la contaminación del medio marino por parte de buques dentro de la zona económica de los Estados, en particular, en aquellas áreas del globo en donde las condiciones climáticas extremas y la presencia del hielo suponen peligros excepcionales para la navegación y la contaminación del medio marino. Como limitantes a la capacidad regulatoria de los Estados se imponen el respeto a la navegación y

${ }^{20}$ Loukacheva, Natalia, The Artic Promise: Legal and Political Autonomy of Greenland and Nunavut, Toronto, Buffalo, London, University of Toronto Press, 2007, p. 122. Acerca de la posibilidad de considerar el Consejo Ártico como una organización internacional, ver: Byers, Michael, International Law and the Arctic, New York, Cambridge University Press, 2013, p. 9.

${ }^{21}$ Nowlan, L., op cit., p. 5.

${ }^{22}$ Rothwell, Donald, The Polar Regions and the Development of International Law, Cambridge, Cambridge University Press, 1996, p. 157.

${ }^{23}$ Convención de las Naciones Unidas sobre el Derecho del Mar, Montego Bay, 10 de diciembre de 1982, 1833 UNTS 3 ['Convemar'].

${ }^{24}$ Brilmayer, Lea \& Klein, Nataly, "Land and Sea: Two Sovereignty Regimes in Search of a Common Denominator", NYU Journal of International Law and Politics, Vol. 33, 2001, p. 732-36.

${ }^{25}$ Hanquin, Xue, Transboundary Damage in International Law, New York, Cambridge University Press, 2003 , p. 193.

${ }^{26}$ Feder, Barnaby, op. cit., p. 787. 
la protección y preservación del medio marino, así como la utilización de los mejores conocimientos científicos disponibles ${ }^{27}$.

Siendo claro el carácter limitado de regulación prevista en la Convemar, en el marco de la OMI se adoptó el Convenio Internacional para la Seguridad de la Vida Humana en el Mar (SOLAS) ${ }^{28}$, el que obliga a los Estados del pabellón a asegurar que en la construcción, el equipamiento y la utilización de buques se apliquen normas compatibles con la seguridad de la navegación. La Regla V del Convenio obliga a los Estados a promover que sus buques recojan, difundan e intercambien datos meteorológicos, incluyendo respecto de la presencia de hielo, y colaboren con los demás Estados Parte para ejecutar medidas que prevengan a los buques contra circunstancias climáticas extremas. La Regla 6 consagra el "Servicio de vigilancia de hielos" y asigna a los Estados Parte la obligación de mantenerlo en funcionamiento. Los esfuerzos de regulación técnica específicos en el Ártico reconocen la importancia de SOLAS para avanzar directrices con vocación de obligatoriedad. Así lo concluye Oysten Jensen tras un exhaustivo análisis acerca de la efectividad de las Directrices de la OMI para los buques que naveguen en aguas polares ${ }^{29}$.

Un instrumento de igual relevancia es el Convenio Internacional para Prevenir la Contaminación de los Buques (MARPOL), adoptado el 2 de noviembre de 1973 en el marco de la OMI. Previo a su entrada en vigor el Convenio fue enmendado por el Protocolo MARPOL de 1978, razón por la que ambos textos nacieron a la vida jurídica como un único instrumento (MARPOL 73/78). Por cuanto el instrumento entraría en vigor el 2 de octubre de 1983, conceptualmente es correcto aludir a MARPOL como un desarrollo del artículo 234 de la Convemar, con la ventaja de que las obligaciones en uno no dependen de la ratificación del otro ${ }^{30}$. Algunos desarrollos posteriores hicieron posible la introducción de enmiendas en forma de anexos técnicos, algunos de los cuales prevén la designación de ciertas áreas marítimas como zonas especiales ${ }^{31}$. Una designación tal encuentra fundamento en las condiciones oceanográficas y ecológicas, así como el tráfico marítimo de la zona, e implica el establecimiento de controles más estrictos y específicos, e incluso la prohibición total de vertimientos. Sin perjuicio de lo anterior, el Océano Ártico no se encuentra entre las zonas especiales y, por tanto, su protección, contra instancias de contaminación accidentales o procedentes de las

${ }^{27}$ Skaridov, Alexander, "Polar Seas", en Nordquist, Myron H., et al. (Eds.), The Stockolm Declaration and Law of the Marine Environment, The Hague, London, New York, Martinus Nijhoff Publishers, 2003, p. 205.

281184 UNTS 3.

29 "The IMO Guidelines for Ships Operating in Arctic Ice-Covered Waters: From Voluntary to Mandatory Tool for Navigational Safety and Environmental Protection?”. Disponible en: http://www.fni. no/pdf/FNI-R0207.pdf.

${ }^{30}$ Vartanov, Raphael V. \& Brodus, James V. (Eds.), The Oceans and Environmental Security: Shared U.S. and Russian Perspectives, Washington D.C., Island Press, 1994, p. 179.

${ }^{31}$ MARPOL es especialmente relevante en la prevención de instancias de contaminación derivadas del transporte marítimo, y por la extensión de su ámbito de aplicación a plataformas flotantes es apto para la protección del medio marino frente a actividades de exploración y explotación de reservas de gas y petróleo. Casper, Kristin N, "Oil and Gas Development in the Arctic: Softening of Ice Demands Hardening of International Law”, Natural Resource Journal, Vol. 49, 2009, p. 852. 
operaciones normales, se realiza mediante mecanismos de protección genéricos de MARPOL y sus anexos técnicos ${ }^{32}$.

\section{Actuaciones específicas por parte de los Estados árticos}

El gran desastre nuclear ocurrido en Chernóbil en 1986 y el desastre del Exxon Valdez de 1989 fueron los principales catalizadores en la concertación de una Estrategia para la Protección Medioambiental del Ártico (AEPS por sus siglas en inglés) ${ }^{33}$. La Estrategia tiene como sustento jurídico la Declaración de Rovaniemi concerniente a la protección del medio ambiente Ártico de 1991 adoptada en la referida Conferencia en Finlandia ${ }^{34}$. La AEPS está estructurada en función del cumplimiento de ciertos objetivos, entre los que se incluyen i) la protección, el desarrollo y restauración del medio ambiente y la utilización sostenible de los recursos naturales, incluyendo su uso por parte de las poblaciones locales e indígenas en el Ártico; ii) el reconocimiento y, en la medida de lo posible, la adaptación y acomodación de las necesidades tradicionales y culturales, así como de los valores y prácticas de las poblaciones indígenas, según sean determinadas por ellas mismas, en relación con la protección del medio ambiente Ártico; y iii) la identificación, reducción y, como meta final, eliminación de la contaminación ${ }^{35}$.

En el análisis del desarrollo del régimen jurídico regional aplicable a la protección ambiental del Océano Ártico es primordial mencionar la compaginación de la AEPS con la creación del Consejo Ártico. Este organismo se estableció en 1996 mediante la Declaración de Ottawa, y aunque no se creó como una organización internacional de derecho internacional público stricto sensu ${ }^{36}$, fue el foro intergubernamental de alto nivel ideado con el propósito de servir de escenario y herramienta para promover la cooperación, coordinación e interacción entre los Estados árticos, con miras a asegurar

\footnotetext{
32 Weidermann, Lilly, op. cit., 117.
}

33 Según lo explica Caulfield, el desastre en Chernóbil (Ucrania) esparció material radiactivo en gran parte del Círculo Ártico con efectos particularmente nocivos en los grupos poblaciones que, como el pueblo Saami, consume alimentos que concentran el elemento químico cesio, entre ellos, el liquen, la carne de reno, los peces y las setas. Ver Caulfield, R. A., "The political, economy and renewable resource management in the Arctic”, Nuttal, Mark y Callaghan, T. V. (Eds.), The Arctic: Environment, People, Policy, Harwood Academic Publishers, Amsterdam, 2000, pp. 492.

34 Arctic Environmental Protection Strategy, Declaration on the Protection of the Arctic Environment, Rovaniemi, Junio 14 de 1991, <http:/www.arctic-council.org/index.php/en/document-archive/category/4founding-documents?download=53:aeps.>, ['Declaración de Rovaniemi']

${ }^{35}$ Declaración de Rovaniemi, pp. 6-8. Donald R. Rothwell puso de presente en 1995 la ausencia histórica de grandes grupos humanos en la región hizo más difícil la generación de una conciencia e identidad frente a los intereses árticos, situación que explicaría en parte la tardía adopción de acciones conjuntas por parte de los Estados árticos. Rothwell, Donald R., "International Law and the Protection of the Arctic Environment”, International and Comparative Law Quarterly, Vol. 44/02 (1995), p. 280.

${ }^{36}$ Bloom, Evan T, "Establishment of the Arctic Council”, The American Journal of International Law, Vol. 93 (2), 1999, p. 721. 
el desarrollo sostenible de la región, así como su protección ambiental ${ }^{37}$. Igualmente, se pensó para servir de foro para la participación de las comunidades indígenas y otros pueblos del ártico en relación con asuntos de interés común ${ }^{38}$.

El Consejo Ártico tiene el mismo patrón de funcionamiento y en sus inicios estuvo compuesto por los mismos grupos de trabajo que la AEPS. Sin embargo, además de disponer de una autonomía mayor que la de la AEPS su marco de acción es más amplio ${ }^{39}$. Tiene como mandatos específicos la implementación de un programa de desarrollo sostenible, así como la divulgación de información y promoción de la educación y el interés en los asuntos propios del Ártico. Entre los principales resultados del Consejo se encuentran el Documento sobre el Marco de Desarrollo Sostenible $e^{40}$ y el Plan de Acción del Ártico para la Eliminación de la Contaminación en el Ártico ${ }^{41}$.

La doctrina expresa con razón que los grupos de trabajo y el consenso, principio rector en la toma de decisiones, constituyen las piedras angulares en el funcionamiento del Consejo Ártico. Esto es cierto, pues el trabajo en el Consejo Ártico progresa y se concreta de forma vertical desde los grupos de trabajo hasta el nivel ministerial, situación que eleva la posibilidad de llegar a decisiones consensuadas en el nivel político. En los grupos de trabajo las decisiones son más detalladas y exhaustivas y propicia la participación de observadores en las discusiones. Los Funcionarios Árticos de Alto Nivel o Senior Arctic Officials (SAO), una figura creada en la AEPS y que se encarga de coordinar, guiar y monitorear las actividades de los órganos subsidiarios del Consejo Ártico, deciden respecto de la remisión de las iniciativas a la Reunión Ministerial una vez que se ha alcanzado el nivel de consenso requerido. Se trata de decisiones cuyo contenido ha sido socializado, conocido, comprendido y discutido por una gran variedad de actores. Por esta razón, las decisiones adoptadas en el Consejo Ártico están revestidas de altísimos niveles de legitimidad ${ }^{42}$.

Como consecuencia de la mayor amplitud de sus funciones, los grupos de trabajo del Consejo Ártico han superado a los existentes en la AEPS.

El Programa de Monitoreo y Evaluación del Ártico (AMAP, por sus siglas en inglés) es responsable por la medición y análisis de los efectos de los contaminantes

37 "Declaration on the Establishment of the Arctic Council", Ottawa, Canada, Sept. 19, 1996. Disponible en: https://oaarchive.arctic-council.org/handle/11374/85. Berkman, Paul A., Environmental Security in the Arctic Ocean: Promoting Co-operation and Preventing Conflict, Royal United Services Institute for Defence and Security Studies, 2010 p. 4.

38 Nuttall, Mark, Protecting the Arctic: Indigenous Peoples and Cultural Survival, Routledge, 1998, p. 37.

39 Weidemann, Lilly, International Governance of the Arctic Marine Environment with Particular Emphasis on High Seas Fisheries, Hamburg, Springer, 2013, p. 49;

${ }^{40}$ Framework Document (Chapteau) for the Sustainable Development, Disponible en: <http://www. arctic-council.org/index.php/en/document-archive/category/4-founding-documents >

${ }^{41}$ Arctic Council Action Plan to Eliminate Pollution of the Arctic, Disponible en: <http://www.arcticcouncil.org/index.php/en/document-archive/category/70-acap-organizational-documents?download=269:acapoverall-strategy-october-2000>.

42 Graczyk, Piotr \& Koivurova, Timo, "The Arctic Council”, en Jensen, Leif C. \& Honneland, Geir, Handbook of the Politic of the Arctic, Northampton, Edward Publishing, 2015, p. 309. 
antropogénicos en el medio ambiente ártico, incluyendo la fauna, la flora y con especial atención en aquellos recursos utilizados por los grupos indígenas ${ }^{43}$. De esta forma, asesora a los Estados Árticos en relación con peligros ambientales derivados o asociados con la contaminación. Por su parte, el Programa de Conservación de la Flora y Fauna Ártica (CAFF, por sus siglas en inglés) es un foro de interacción entre científicos, pueblos indígenas, grupos conservacionistas para el intercambio de información para el análisis de especies y hábitats compartidos y la cooperación en aras de identificar formas más eficaces de utilización sostenible, conservación y preservación de la diversidad ártica ${ }^{44}$. El Grupo de Trabajo sobre Prevención de Emergencias, Preparación y Respuesta (EPPR, por sus siglas en inglés) tiene como propósito el diseño de metodologías y directrices de análisis de riesgo, ejercicios de respuesta y entrenamiento frente a accidentes en el mar o en la tierra con efectos negativos en el medio ambiente ártico ${ }^{45}$.

Un referente contemporáneo atinente a la acción y aporte efectivo de los grupos de trabajo lo constituye el denominado Environmentally Safe Management of Stocks of Obsolete and Probibited Pesticides in Russia, proyecto desarrollado por los Estados árticos en respuesta a la información presentada por AMAP en el Consejo Ártico, e implementado por el Programa de Acción contra los Contaminantes Árticos (ACAP, por sus siglas en inglés) y, en particular, por su Grupo Experto sobre Pesticidas Obsoletos. En la medida en que varios sistemas fluviales de la Federación Rusa llegan al Océano Ártico, se observó con especial preocupación la constatación de que varias decenas de miles de toneladas de pesticidas prohibidos se almacenaban en ese Estado. Por esta razón, ACAP implementó el proyecto en los territorios del norte de Rusia desde el $2001^{46}$.

Un importante aporte del EPPR está constituido por el denominado Agreement on Cooperation on Marine Oil Pollution Preparedness and Response in the Arctic, acuerdo de carácter recomendatorio que sin modificar las obligaciones de los Estados conforme al derecho internacional, consagra directrices de carácter procedimental para hacer efectiva la cooperación, coordinación y asistencia mutua frente a eventuales derrames de petróleo en el Ártico ${ }^{47}$. Los autores destacan que el Acuerdo enlista las obligaciones jurídicamente vinculantes en el nivel bilateral y multilateral entre los Estados Árticos, aspecto que eleva las posibilidades de que las directrices tengan un efecto útil.

Uno de los aportes más importantes del grupo de Protección del Medio Ambiente Marino Ártico (PAME, por sus siglas en inglés) es el reporte denominado "Evaluación

43 Entre otros reportes, ver: “AMAP, 2011.Artic Pollution 2011: Mercury”. Disponible en: http:// www.amap.no/documents/doc/arctic-pollution-2011-mercury/89.

44 Weidemann, op. cit., p. 52.

45 De obligatoria mención a este respecto en este punto es el llamado que en el 2011 la reunión ministerial le hizo al EPPR para que, en cooperación con otros grupos, desarrollara recomendaciones y mejores prácticas para la prevención de la contaminación marina y para que entregara sus resultados en un término de 2 años. Vanderzwaag, David L., "The Arctic Council at 15 Years: Edging Forward in a Sea of Governance Challenges”, German Yearbook of International Law, Vol. 54, 2011, p. 292.

${ }^{46}$ Disponible en: http://www.arctic-council.org/index.php/en/about-us/working-groups/51-acap.

47 Disponible en: http://arctic-council.org/eppr/wp-content/uploads/2014/03/EDOCS3211-v3-2016_02_12_APPENDIX_IV_OPERATIONAL_GUIDELINES.pdf. 
de 2009 sobre el Transporte Marino en el Ártico" (AMSA, por sus siglas en inglés), el que analiza de forma exhaustiva y crítica la adecuación de las recomendaciones y directrices aplicables para ese momento. AMSA incorporó 17 recomendaciones entre las que se incluyeron el apoyo a la aplicación vinculante y obligatoria de algunas directrices, entre ellas las "Directrices para buques que navegan en aguas polares" de la $\mathrm{IMO}^{48}$; la consideración de la armonización de las normativas domésticas de los Estados en relación con el transporte marítimo en el Ártico; y el análisis sobre la necesidad de designación de ciertas áreas del Océano Ártico como zonas especiales. Según lo describe David L. Vanderzwaag, AMSA tuvo importantes consecuencias tanto en el nivel normativo como institucional, entre los que se incluyen el actual Código Polar de la OMI, el establecimiento de la Comisión Hidrográfica Regional Ártica en el marco de la Organización Hidrográfica Internacional, un trabajo conjunto entre AMAP, CAFF y SDWG para la identificación de áreas de importancia ecológica y cultural preponderante en el Ártico ${ }^{49}$. Las labores de PAME también se han dirigido a la revisión de las "Directrices sobre actividades Offshore de Petróleo y Gas”, adoptadas en el Consejo Ártico en el 2009, inter alia, con el propósito de promover la adopción de principios comunes en las legislaciones de los Estados miembros, así como el establecimientos de procedimientos para la evaluación de los impactos medioambientales de los proyectos y su incidencia en los grupos indígenas ${ }^{50}$.

En este marco es igualmente posible verificar la existencia de organizaciones no gubernamentales entre las que destacamos el Consejo Nórdico o Consejo Saami, el que tiene estatus de participación permanente en el Consejo del Ártico y representa a los pueblos indígenas del Ártico, en particular, el pueblo Saami ${ }^{51}$. Asimismo, se resalta la labor del Comité Científico Internacional del Ártico (IASC, por sus siglas en inglés), que tiene como propósito promover y facilitar la cooperación en todos los aspectos de la investigación científica entre los Estados árticos ${ }^{52}$. Finalmente, la Conferencia Permanente de Parlamentarios de la Región Ártica, de naturaleza no gubernamental, reúne delegados designados por los parlamentos nacionales de los Estados árticos, quienes interactúan con delegados de los pueblos indígenas y designados del Parlamento Europeo ${ }^{53}$.

Sin perjuicio de lo anterior, se identifican importantes elaboraciones doctrinales que elevan interrogantes acerca de la capacidad del Consejo Ártico y del sistema legal vigente para dar cuenta de las nuevas y urgentes amenazas generadas por el cambio

\footnotetext{
${ }^{48}$ Resolución A.1024 (26), adoptada el 2 de diciembre de 2009. Disponible en: http://www.imo.org/ es/MediaCentre/HotTopics/polar/Documents/A\%2026-Res.1024.pdf.

49 Vanderzwaag, David L, op. cit., p. 294.

50 “Arctic Council, Arctic Offshore Oil and Gas Guidelines, 29 April 2009”. Disponible en: https:// www.govmin.gl/images/stories/petroleum/Arctic_Offshore_Oil_and_Gas_Guidelines_2009.pdf.

${ }^{51}$ Saami Council, "About Saami Council”, [website] <http://www.saamicouncil.net/?deptid=2178.>, últimavisita: 09/01/2015.

52 International Arctic Science Committee, “About IASC”, [website]: <http://www.iasc.info/home/ iasc. >, últimavisita: 09/01/2015.

${ }^{53}$ Nowlan, L., op. cit., pp. 7.
} 
climático. A este respecto, Vanderzwaag se detiene con especial atención en los grupos de trabajo del Consejo Ártico para destacar la flexibilidad y capacidad de dinamismo de este órgano de concertación política. En su consideración, el del Consejo Ártico es un "trabajo en progreso" y así deben valorarse sus logros y su capacidad para dar cuenta de los nuevos retos, especialmente los que impone el cambio climático ${ }^{54}$.

En relación con la capacidad de acción del Consejo Ártico es importante tener presente que, mediante la Declaración de la Reunión Ministerial celebrada en Nuuk en mayo de 2011, se dotó al Consejo de una Secretaría permanente. El establecimiento de un órgano de carácter permanente ha propiciado la producción de una nueva doctrina que caracteriza al Consejo Ártico como una verdadera organización internacional, comparándola con otras que como la Organización para la Seguridad y la Cooperación en Europa (OSCE, por siglas en inglés) dispone de una personalidad internacional derivada no obstante su nacimiento mediante una declaración intergubernamental y no de un tratado ${ }^{55}$. De otra parte, la negociación y adopción del "Acuerdo sobre Búsqueda y Rescate Aeronáutica en el Ártico” en el marco del Consejo Ártico invita a reconsiderar las premisas tradicionales que le negaban al Consejo la capacidad de propiciar obligaciones jurídicamente vinculantes ${ }^{56}$.

\section{Discusiones acerca de la necesidad de una regulación diferenciada}

Barnaby J. Feder expresó sus dudas respecto de la suficiencia del régimen general del derecho del mar aludiendo a las características medioambientales únicas del Ártico, especialmente al mantenimiento de la capa de hielo como requisito sine qua non para la conservación de esas condiciones ${ }^{57}$. Por su parte, Brooks Yeager y Robert Huebert ${ }^{58}$ enfatizan las deficiencias de una protección fundamentada en la Convemar o en instrumentos de carácter no vinculante y ven en un Tratado Ártico la única posibilidad para generar mecanismos de ejecución efectivos. Estos autores señalan que un tratado que aborde el asunto a partir de las necesidades del ecosistema se justifica por la naturaleza interrelacionada de los desafíos, entre los que se incluyen, de una parte, el manejo del creciente tránsito marítimo y el desarrollo en materia de petróleo y gas, y de la otra, la conservación de las pesquerías, los mamíferos marinos, las aves marinas y el hábitat ${ }^{59}$.

\footnotetext{
${ }^{54}$ Vanderzwaag, David L., "The Arctic Council at 15 Years: Edging Forward in a Sea of Governance Challenges”, German Yearbook of International Law, Vol. 54, 2011, p. 314.

55 "Final Act of the Conference on Security and Cooperation in Europe", 1975, 14 ILM 1292.

${ }^{56}$ Byers Michel, op. cit., p. 9.

${ }^{57}$ Feder, B, op. cit., pp. 786-7.

${ }^{58}$ Yeager, B. y Huebert, R., "A New Sea: The Need for a Regional Agreement on Management and Conservation of the Arctic Marine Environment", 2008, <http://www.arcticgovernance.org/a-new-sea-the-need-for-a-regionalagreement-on-management-and-conservation-of-the-arctic-marine-environment.4627538-137743. html>, pp. 28 .

59 Ibid. Ver también: Young, O., "Whither the Arctic? Conflict or Cooperation in the Circumpolar North”, Polar Record, Vol. 45/1 2009, pp. 73-74.; Borgerson, S., "Arctic Meltdown: The Economic and Security Implications of Global Warming”, Foreign Affairs, 2008, <http://www.foreignaffairs.com/articles/63222/ scott-g-borgerson/arctic-meltdown>, pp. 63, 71-77.
} 
En esta misma línea, Linda Nowlan alude a tres razones que justifican la necesidad, pertinencia y procedencia de un "Tratado Ártico", a saber: i) la importancia del Ártico; ii) la gravedad de las amenazas medioambientales que enfrenta el Ártico; iii) la ausencia de un régimen omnicomprensivo para abordar esas amenazas. En su consideración, un "Tratado Ártico" aportaría cuantitativa y cualitativamente a las obligaciones de los Estados en relación con las amenazas que afectan al Ártico, al tiempo que facilitaría el establecimiento de programas y calendarios basados en metas específicas. Asimismo, Nowlan expresa que la mayor preocupación de un modelo basado en la cooperación y en normas de carácter no vinculante es que su cumplimiento está sujeto a los cambios en la posición política de los gobiernos, preocupación subsanable con un tratado que obligue al Estado in toto ${ }^{60}$.

Los contradictores de la precitada posición resaltan las dificultades inherentes a la negociación de un tratado y la ulterior obtención del consentimiento en relación con asuntos económicamente costosos. Hans Hertell expresó a este respecto que las variables geopolíticas más importantes a tener en cuenta en este campo deben ser las preocupaciones por el medio ambiente ártico y la explotación ordenada y sostenible de sus recursos naturales ${ }^{61}$. De esta forma, los partidarios de un Tratado Ártico observan en el cambio climático y el correlativo acceso a una parte antes inaccesible de los recursos árticos, los puntos de inflexión hacia su celebración ${ }^{62}$.

Sin perjuicio de las consideraciones precedentes, corresponde destacar que entre quienes abogan por un régimen legal diferenciado existe discrepancia con la forma que ese régimen debería adoptar. Verhaag sostiene que el Tratado Ártico a celebrar debería emular el Sistema Antártico ${ }^{63}$. Esta es sin embargo una posición altamente cuestionada y sus partidarios son cada vez menos. Por ejemplo, Davor Vidas distingue uno y otro espacio en función del estado de las reclamaciones territoriales, la histórica presencia militar y la continua explotación de los recursos naturales árticos ${ }^{64}$.

Otros grupos de autores consideran más conveniente y eficaz hacer un mejor uso de los instrumentos y normativas actuales. En este grupo se encuentra Hans Corell, quien señaló que un Tratado Ártico resultaría innecesario y llamó la atención para que se hiciera un mayor uso de la Convemar, sin perjuicio de un análisis de necesidades y escenarios de fortalecimiento ${ }^{65}$.

${ }^{60}$ Nowlan, L., op. cit., pp. 59.

${ }^{61}$ Hertell, H., “Arctic Melt: The Tipping Point for an Arctic Treaty”, Georgetown International Environmental Law Review, 2008-2009, pp. 582-3.

${ }^{62}$ Hertell, H., op. cit., p. 583; Koivurova, T y VanderZwaag, D., op. cit., pp. 180.

${ }^{63}$ Verhaag, M., "It is Not Too Late: The Need for a Comprehensive International Treaty to Protect the Arctic Environment”, Georgetown International Environmental Law Review, 2003, pp. 555, 578.

${ }^{64}$ Vidas, D., “The Polar Marine Environment in Regional Cooperation”, enVidas, D. (Ed.), Protecting the Polar Marine Environment: Law and Policy for Pollution Prevention, Cambridge University Press, 2000, pp. 78,101 .

${ }^{65}$ Corell, H., Reflection on the Possibilities and Limitations of a Binding Legal Regime for the Arctic, 2007, disponibleen<http://www.havc.se/res/SelectedMaterial/20070604corellarcticlegalregenvpolicy1.pdf>, pp. 321-322. 
Para autores como Christopher C. Joyner, la articulación de un régimen normativo específico y, en particular, un Tratado Ártico, parece poco probable debido a la geografía y las políticas divergentes de los Estados árticos ${ }^{66}$. Joyner defiende la aplicación de la Convención al Océano Ártico, destacando entre otros que este régimen ha sido el fundamento del ulterior desarrollo normativo en esta zona ${ }^{67}$. En particular, señala que son nueve las dimensiones que la Convemar permite abordar en relación con el Océano Ártico, estas pueden ser agrupadas a su turno en las siguientes áreas: i) jurisdicción territorial; ii) administración de las pesquerías; iii) conservación de recursos; iv) prevención de contaminación; v) medidas en relación con el vertimiento de desechos y desperdicios; vi) regulación del comercio internacional; vii) manejo de procesos globales; viii) paz y control de armas; y ix) actividades criminales en el mar.

En el nivel institucional ártico, PAME analizó la efectividad de las actuales reglas de protección de medio ambiente aplicables al Ártico y concluyó que los instrumentos vigentes otorgan una base legal adecuada para su protección sin que sea posible verificar ninguna necesidad que justifique el desarrollo de nuevos instrumentos ${ }^{68}$. Más de 12 años después y sobre la base de un reconocimiento público respecto de las consecuencias del cambio climático en el Ártico, la Declaración de la Conferencia Ilulissat, de 28 de mayo 2008, ratificó la anterior posición ${ }^{69}$.

En la Declaración de Ilulissat los Estados árticos declararon que actualmente existe un extenso régimen legal mediante el cual pueden abordarse con suficiencia la protección del medio ambiente marino ártico, incluyendo las áreas cubiertas de hielo. Los Estados reconocieron los efectos especialmente graves que los accidentes derivados de la navegación han tenido en el medio ambiente Ártico y en las formas de vida de los habitantes de la zona. Sin embargo, estimaron que las medidas a nivel nacional y mediante la cooperación internacional resultarían suficientes para garantizar la protección y preservación de este frágil ambiente marino. Sin perjuicio de lo anterior, se expresó la intención de trabajar con las organizaciones internacionales concernidas, entre ellas, la Organización Marítima Internacional (OMI), para fortalecer las medidas existentes y desarrollar nuevas estrategias que aumenten la seguridad en la navegación marítima y prevengan o reduzcan el riesgo de contaminación emanada de los buques.

${ }^{66}$ Joyner, C., "The Legal Regime for the Arctic Ocean”, Journal of Transnational Law E Policy, Vol. 18/2, 2008-2009, pp. 199.

${ }^{67}$ Ibidem.

68 PAME, Report to the Third Ministerial Conference on the Protection of the Arctic Environment (1996, Inuvik, Canada), disponible en: <http://arcticportal.org/pame/pame-document-library/pame-1996-report.pdf.>, p. 83.

${ }^{69}$ La Declaración Ilulissat, Conferencia del Océano Ártico, disponible en: <http://www.oceanlaw.org/ downloads/arctic/Ilulissat_Declaration.pdf.> , ['Declaración Ilulissat']. 


\section{CONSIDERACIONES FINALES}

Los partidarios de una normativa convencional vinculante y específica para el Océano Ártico observan con especial preocupación que la inclusión de estándares de protección en cualquier otro tipo de instrumento desconocería las especiales condiciones del medio ambiente ártico y estaría sometida a la discrecionalidad del gobierno de turno.

Acerca del particular, es preciso señalar que el régimen genérico previsto en la Convemar contrasta con las múltiples y diversas manifestaciones de la fragilidad y vulnerabilidad del medio ambiente ártico ${ }^{70}$. Siendo esto cierto, también lo es que los Estados Árticos se han mostrado conscientes de la especial fragilidad del medio ambiente ártico. El cuestionamiento es si la normativa disponible ofrece garantías suficientes de protección.

En la posición de los autores, la principal herramienta frente a los problemas ambientales del Océano Ártico está constituida por la cooperación entre los Estados de esta parte del globo. Aunque la cooperación halla una fuente normativa vinculante en los artículos 118 y 119 de la Convemar, los Estados árticos se han valido de ella de forma extensiva para generar verdaderas instituciones representativas que sobreviven a los gobiernos de turno y que se proyectan como instancias efectivas frente a la comunidad internacional, tal es el caso del Consejo Ártico, el que goza del dinamismo y legitimidad decisoria producto de la labor de sus grupos de trabajo y ha evolucionado hasta disponer de órganos permanentes, y promover la celebración de tratados jurídicamente vinculantes.

En este contexto corresponde destacar que entre el 11 y el 15 de mayo de 2015, en el marco de la sexagésimo octava sesión del Comité para la Protección del Medio Ambiente Marino del Ártico de la OMI, se adoptaron las disposiciones medioambientales del Código sobre Navegación en Aguas Polares, también conocido como Código Polar de la OMI. Se espera que la totalidad del Código entre en vigor el 1 de enero de 2017. Según se expresa en el portal web de la OMI, el propósito es que las disposiciones del Código sean legalmente vinculantes bajo SOLAS y MARPOL ${ }^{71}$. El Consejo Ártico emitió un comunicado manifestando la importancia de la adopción de estas nuevas disposiciones, las que complementan la normativa existente de la OMI al prever restricciones adicionales que buscan abordar las amenazas específicas que generan los buques comerciales en los dos polos ${ }^{72}$.

La posición de los autores además resalta la importancia de los instrumentos convencionales generales en materia ambiental, en lugar de obviarlos mediante la aceptación de la negociación de novo de un "Tratado Ártico". De esta forma, contribuye a evitar incurrir en los fenómenos de duplicidad normativa y falta de coordinación de los que adolecen otros sistemas globales de protección. Esta aproximación reconoce que los fenómenos

${ }^{70}$ Anisimov, O. y Vaughan, D. (Coord), "Polar regions (Arctic and Antarctic)" en Parry, M. et al. (Eds), Climate Change 2007: Impacts, Adaptation and Vulnerability, Cambridge University Press, Cambridge, 2007, pp. 653-685.

71 "Adoption of an international code of safety for ships operating in polar waters (Polar Code)". Disponible en: http://www.imo.org/en/MediaCentre/HotTopics/polar/Pages/default.aspx.

${ }^{72}$ International Convention for the Prevention of Pollution from Ships, 12 ILM 1319 (1973). 
que con mayor rigor se proyectan sobre el Ártico, inter alia, el calentamiento global, resultan de actuaciones estatales en la totalidad del globo.

\section{BiBLIOGRAFÍA}

\section{Doctrina}

Anisimov, O. y Vaughan, D. (Coord), "Polar regions (Arctic and Antarctic)", en Parry, M. et al. (Eds.), Climate Change 2007: Impacts, Adaptation and Vulnerability, Cambridge University Press, Cambridge, 2007.

Baker, B., "Filing an Arctic Gap: Legal and Regulatory Possibilities for Canadian-U.S. Cooperation in the Beufort Sea", Vermont Law Review, Vol. 34, 2010.

Berkman, Paul A., Environmental Security in the Arctic Ocean: Promoting Co-operation and Preventing Conflict, Royal United Services Institute for Defence and Security Studies, 2010 p. 4.

Bloom, Evan T, "Establishment of the Arctic Council", The American Journal of International Law, Vol. 93 (2), 1999.

Brilmayer, Lea \& Klein, Nataly, "Land and Sea: Two Sovereignty Regimes in Search of a Common Denominator", NYU Journal of International Law and Politics, Vol. 33, 2001.

Byers, Michael, International Law and the Arctic, New York, Cambridge University Press, 2013.

Caflish, L., "Les zones maritimes sous jurisdictionnationale, leurs limites et leur délimitation", en Bardonnet, D. y Virally M., Le nouveau droitinternational de la mer, Pedine, Paris, 1983.

Casper, Kristin N, "Oil and Gas Development in the Arctic: Softening of Ice Demands Hardening of International Law", Natural Resource Journal, Vol. 49, 2009.

Caulfield, R. A., "The political, economy and renewable resource management in the Arctic", Nuttal, Mark y Callaghan, T. V. (Eds.), The Arctic: Environment, People, Policy, Harwood Academic Publishers, Amsterdam, 2000.

Chaturvedi, Sanjay, The Polar Regions: A Political Geography, Wiley, 1996.

Colacrai de Trevisan, M., El Ártico y la Antártida. Su rol en las relaciones internacionales. Su relevancia desde la perspectiva ambiental, Centro de Estudios en Relaciones Internacionales de Rosario (CERIR) y Centro de Estudios Canadienses de Rosario (CECAR), 1998.

Conley, H., Arctic Economics in the $21^{\text {st }}$ Century. The Benefits and Costs of Cold, Center of Strategic \& International Studies, Washington D.C., 2013.

Corell, H, Reflection on the Possibilities and Limitations of a Binding Legal Regime for the Arctic, 2007.

Feder, Barnaby., 'A Legal Regime for the Arctic', Ecology Law Quarterly, Vol. 6/4, 1978.

GraczyK, Piotr \& Koivurova, Timo, "The Arctic Council”, en Jensen, Leif C. \& Honneland, Geir, Handbook of the Politic of the Arctic, Northampton, Edward Publishing, 2015.

Hanquin, Xue, Transboundary Damage in International Law, New York, Cambridge University Press, 2003.

Hertell, H., "Arctic Melt: The Tipping Point for an Arctic Treaty", Georgetown International Environmental Law Review, 2008-2009.

Holmes, S., "Breaking the Ice: Emerging Legal Issues in Arctic Sovereignty", Chicago Journal of International Law, Vol. 9, 2008.

Joyner, C., "The Legal Regime for the Arctic Ocean", Journal of Transnational Law E Policy, Vol. 18/2, 2008-2009.

KWIATKOWSKA, Barbara., "Submissions to the UN Commission on the Limits of the Continental Shelf: The Practice of Developing States in Cases of Disputed and Unresolved Maritime 
Boundary Delimitations or Other Land or Maritime Disputes. Part One”, The International Journal of Marine and Coastal Law.

Koivurova, T. y VanderZwaag, D., "The Arctic Council at 10 Years: Retrospect and Prospects", University of British Columbia Law Review, Vol.40/1, 2007.

Lakhtine, W., "Rights Over the Arctic", AMYI, Vol. 24, 1930.

Loukacheva, Natalia, The Artic Promise: Legal and Political Autonomy of Greenland and Nunavut, Toronto, Buffalo, London, University of Toronto Press, 2007.

MArqués, E., "La condición jurídica del Ártico y la Antártica: un asunto pendiente en la agenda jurídico-política de las relaciones internacionales contemporáneas”, Revista de Relaciones Internacionales de la UNAM, $\mathrm{N}^{\circ}$ 107, Mayo-Agosto, 2010.

Matz-LüCK, N., "Planting the Flag in the Arctic Waters: Russia's Claim to the North Pole", Gôttingen Journal of International Law, Vol. 2, 2009.

MineK, R, "Enviromental Protection of the Arctic", <http://www.amap.no/documents/download/67>

Nielsen, T., "Floating Nuclear Plants in the Siberian Arctic?", World Wildlife Foundation, Arctic Bulletin 4.00, 2000.

Nowlan, L., Arctic legal regime for Environmental Protection, International Union for Conservation of Nature and Natural Resources, Gland, Cambridge and Bonn, 2001.

Nuttall, Mark, Protecting the Arctic: Indigenous Peoples and Cultural Survival, Routledge, 1998.

Riddell-Dixon, Elizabeth: "Canada and Arctic Politics: The Continental Shelf Extension", Ocean Development and International Law, Vol. 39 (4), 2008.

Rothwell, D., "International Law and the Protection of the Arctic Environment", International and Comparative Law Quarterly, Vol. 44/02, 1995.

Rothwell, Donald, The Polar Regions and the Development of International Law, Cambridge, Cambridge University Press, 1996.

Rothwell, D. y Joyner, C., "Domestic Perspectives and Regulations in Protecting the Polar Marine Environment: Australia, Canada and the United States”, en Vidas, D.(Ed.), Protecting the Polar Marine Environment: Law and Policy for Pollution Prevention, Cambridge University Press, 2000.

Shusterich, K., "International Jurisdictional Issues in the Arctic Ocean", Ocean Development E International Law, Vol. 14/3, 1984.

SKaridov, Alexander, "Polr Seas", en Nordquist, Myron H., et al. (Eds.), The Stockolmk Declaration and Law of the Marine Environment, The Hague, London, New York, Martinus Nijhoff Publishers, 2003.

Stepens T. \& VanderZwaag David L. (Eds.), Polar Oceans Governance in an Era of Environmental Change, Northampton, Edward Elgar, 2014.

VanderzWaag, David L., "The Arctic Council at 15 Years: Edging Forward in a Sea of Governance Challenges", German Yearbook of International Law, Vol. 54, 2011.

VerhaAg, M., "It is Not Too Late: The Need for a Comprehensive International Treaty to Protect the Arctic Environment", Georgetown International Environmental Law Review, 2003.

VIDAS, D., "The Polar Marine Environment in Regional Cooperation”, en Vidas, D. (Ed.), Protecting the Polar Marine Environment: Law and Policy for Pollution Prevention, Cambridge University Press, 2000.

Weidemann, Lilly, International Governance of the Arctic Marine Environment with Particular Emphasis on High Seas Fisheries, Hamburg, Springer, 2013.

Wolfe, L. y Hoult, D., "Effects of Oil under sea ice”, Journal of Glaciology, Vol. 13/69, 1974.

Yeager, B. y Huebert, R., A New Sea: The Need for a Regional Agreement on Management and Conservation of the Arctic Marine Environment, 2008.

Young, O., "Whither the Arctic? Conflict or Cooperation in the Circumpolar North", Polar Record, Vol. 45/1, 2009. 


\section{Documentos de Naciones Unidas}

ACUERDO para promover el cumplimiento de las medidas internacionales de conservación y ordenación por los Buques Pesqueros que pescan en Alta Mar, 24 de noviembre de 1993, 33 I.L.M. 968.

\section{Otros}

'ARCTIC Council Action Plan to Eliminate Pollution of the Arctic (ACAP), Disponible en: <http:// www.arctic-council.org/index.php/en/document-archive/category/70-acap-organizational-documents? download=269: acap-overall-strategy-october-2000.>

ARCTIC Environmental Protection Strategy, Declaration on the Protection of the Arctic Environment, Rovaniemi, Junio 14 de 1991, <http://www.arctic-council.org/index.php/en/document-archive/ category/4-founding-documents? download=53:aeps. >

Convención de las Naciones Unidas sobre el Derecho del Mar, Montego Bay, 10 de diciembre de 1982, 1833 UNTS 3; 21 ILM 1261 (1982)

La Declaración Ilulissat, Conferencia del Océano Ártico, disponible en: <http://www.oceanlaw. org/downloads/arctic/Ilulissat_Declaration.pdf.>

Declaration on the Establishment of the Arctic Council”, Ottawa, Canada, Sept. 19, 1996. Disponible en: https://oaarchive.arctic-council.org/handle/11374/85

International Arctic Science Committee, “About IASC”, [website]: <http://www.iasc.info/ home/iasc. >, última visita: 09/01/2015

Protocolo al Tratado Antártico sobre protección del medio ambiente, Madrid, 4 de octubre de 1991, 30 ILM 1455 (1991)

PAME, Working Group on the Protection of the Arctic Marine Environment, Report to the Third Ministerial Conference on the Protection of the Arctic Environment (1996, Inuvik, Canada), disponibleen<http://arcticportal.org/pame/pame-document-library/pame-1996-report.pdf.>

SAAmI Council, "About Saami Council”, [website] <http://www.saamicouncil.net/?deptid=2178.>, última visita: 09/01/2015

Tratado Antártico, Washington, 1 de diciembre de 1959, 12 UST 794; 402 UNTS 71; 19 ILM 860 (1980)

THE Arctic Council Regional Programme of Action for the Protection of the Arctic Marine Environment from Lad-based Activities (RPA), Disponible en: <http://www.arctic-council.org/index.php/ en/document-archive/category/235-5-oceans?download=867:rpa > 